\title{
Chemical Pathology of Chemerin and Its Link to Obesity and Type 2 Diabetes Mellitus: A Review
}

\author{
Ekpe E. E. ${ }^{1}$, Ekpe V. E. ${ }^{2}$ \\ ${ }^{1}$ Department of Chemical Pathology, University of Calabar Teaching Hospital, Calabar, Nigeria \\ ${ }^{2}$ Dornsife School of Public Health, Drexel University, Philadelphia, USA
}

\section{Email address:}

lawsonekpe2002@yahoo.com (Ekpe E. E.)

\section{To cite this article:}

Ekpe E. E., Ekpe V. E. Chemical Pathology of Chemerin and Its Link to Obesity and Type 2 Diabetes Mellitus: A Review. Biochemistry and Molecular Biology. Vol. 2, No. 4, 2017, pp. 37-39. doi: 10.11648/j.bmb.20170204.11

Received: April 29, 2017; Accepted: June 1, 2017; Published: July 14, 2017

\begin{abstract}
Adipocytokines have been widely recognized in scientific research in recent times because of their varied functions and roles in the body. One of such recognized adipocytokines, is chemerin. A review of chemerin, is presented in this paper with a view to assessing the pathophysiology involved in obesity and type 2 diabetes mellitus. Published literatures were analyzed with the aim of assessing the correlation of chemerin in relation to obesity and diabetes mellitus. Searched literatures and journals from various researchers conclude with the evidence of increased levels of chemerin in type 2 diabetes mellitus patients which might play a major role in the pathogenesis of obesity and type 2 diabetes mellitus. Further studies are needed to understand the factors correlating chemerin levels to obesity and type 2 diabetesmellitus. This could enhance pharmacologic management of diabetic and obese patients.
\end{abstract}

Keywords: Chemerin, Diabetes Mellitus, Obesity, Adipocytokines

\section{Introduction}

Adipokines are cell signaling proteins that are usually secreted by the adipose tissues. Leptin was the first adipokine discovered in 1994 [1]. Several other adipokines have been discovered in man viz: adiponectin, apelin, chemerin, interleukins, visfatin, retinol binding protein (RBP- 4), plasminogen activator inhibitor (PAI-1). Adipocytokines are usually either inflammatory mediators (e.g interleukins 6, interleukins 8), angiogegenic proteins (e.g VEGF) and/or metabolic regulators (e.g. adiponectin, leptin) [2]. Chemerin, also known as retinoic acid reactor responder protein 2 (RARRES 2) is an adipokinewhich is encoded by the RARRES 2 gene 1, 2, 3. It is also called Tazarotene-induced gene (TIG 2). Chemerin, a recently discovered protein is an adipocytokine, and has multiple roles in obesity and related complications. It is known that chemerin is a proinflammatory plasma protein that binds to chemerin receptor 23 (Chem $\mathrm{R} 23$ ) on the macrophage and plasmacytoid dendritic cells, where it promotes chemotaxis [3, 4].

It is produced from its precursor protein, prochemerin, which is $18 \mathrm{kDa}$ molecule. This pro-chemerin is converted to chemerin by the cleavage of a $\mathrm{COOH}$-terminal peptide involving serine proteases of the coagulation and inflammation cascades. In this regard, 6 amino acid peptides is cleaved off at its $\mathrm{C}$-terminal end by a serine protease and this produces the active chemerin which is a 137 amino acid protein. This activechemerin binds to $\mathrm{G}$ protein coupled receptor (GPCR), Chem R23 which is usually expressed on macrophage and induces cell migration. Chemerin was first described as a chemo-attractant and it is among the newly discovered adipokines. It is an adipokine with plasma and serum concentration of between 3 and $4.4 \mathrm{~nm}$ in man and it can be assayed by enzyme-linked immunosorbent assay (ELISA). It was discovered by reverse pharmacology [3, 4, 5]. This review focuses on the adipocytokine, chemerin and its role in diabetes mellitus.

\section{Clinical Corrrelation of Chemerin and Obesity and Diabates Mellitus}

It is a protein with large scope and has been liked to insulin-resistance and metabolic syndrome. In recent times, the adipose tissue is increasingly being recognized as an active endocrine organ $[6,7]$. This is so, as many molecules such as vaspin, omentin, adiponectin and a host of other 
molecules are derived from the adipocytes $[8,9]$. Chemerin has been studied by various researchers and it is identified as having a multi-factorial role in the metabolism of lipid and glucose. Studies done by Yang $M$ et al showed that elevated levels of chemerin were noted in newly diagnosed diabetics $(P<0.1)$ compared to controls and previously diagnosed diabetes mellitus. The chemerin levels in newly diagnosed diabetics had an area under the care (AUC) of 0.963 using a receiver operator characteristics (ROC) analysis. Hence chemerin was elevated in pre-diabetic states [10].

Ernest MC et al suggested that chemerin levels are elevated in patients with obesity and type 2 diabetes mellitus [11]. Another study done by Coinmbra et al confirm that circulating concentrations are increased in every patients with elderly type 2 diabetes mellitus [12]. In another study, $\mathrm{Du} \mathrm{J}$ et al assessed the levels of chemerinin diabetic retinopathy patients of type $2 \mathrm{DM}$ patients. They studied 60 type 2 diabetes mellitus (DM) patients and 20 healthy subjects (control group). Using linear regression analysis, the correlation between chemerin and other variables were determined. Statistical analysis showed that the levels of chemerin were significantly increased in patients with retinopathy when compared to the control group [5]. Again, Yang $M$ et al studied the relationship between plasma levels of chemerin in newly diagnosed type 2DM patients with hypertension. Summarily, they found that plasma levels of chemerin were found to be markedly increased in patients with type 2DM with hypertension when compared to patients with type $2 \mathrm{DM}$ and normal controls $(P<0.01)$. Their work supported the claim that chemerin plays a role in the pathogenesis insulin resistance, obesity, and metabolic syndrome [12].

Increased level of chemerin have been hypothesized as a factor in the development of type 2DMas a consequence of dysregulation of the key physiological processes regulated bythis adipokine. It is also proposed as a guide for future research on the role of this adipokine in mediating obesity and the development of type 2 diabetes mellitus [13]. In a Chinese survey, a study to assess the rationship between chemerin and metabolic syndrome components (in this case, chemerin with blood glucose) was done. A total of 30 patients with metabolic syndrome and 30 controls were studied. The researchers found that levels of plasma chemerin was higher in the metabolic syndrome group than the control group $(97.6 \pm 6.49$ vs $70.26 \pm 6.97, P<0.05)$. The plasma chemerin level was poorly correlated with fasting plasma glucose $(\mathrm{r}=0.613, p<0.05)$. Hence, they concluded that plasma chemerin levels correlated with obesity, suggesting it may play a role in metabolic syndrome and alsotype 2 diabetes mellitus [14]. In comparing 76 type 2 diabetes mellitus patients and 76 normal controls, blood glucose and serumchemerin levels were higher in females and obese individualsthan in men and subjects with normal weight. Hence, serumchemerin is correlated with insulin levels and it suggests a role played in the pathophysiology of obesity and metabolic syndrome [15]. Tan et al conducted a cross sectional study on 225 obese children (101 had metabolic syndrome, 124 without metabolic syndrome), and compared with 119 lean controls. Their results showed that chemerin levels were significantly higher in obese children especially those with metabolic syndrome $(p<0.05)$ independent of other well known risk factors. Chemerin was a significant predictor of metabolic syndrome in children. They concluded chemerinis regulated by weight status and seems to be correlated with metabolic syndrome [16]. Li et al conducted a meta analysis involving various researchers linking chemerin with metabolic syndrome, obesity and diabetes mellitus and they concluded that chemerin in obesity/metabolic syndrome may be positively correlated with insulin resistance. Chemerin was found to play a role in pathophysiology of obesity and metabolic syndrome [17].

On the contrary, Robert et al assessed the ability of chemerin levels in prediction of type 2 diabetes mellitus and they found that in the 440 participants that they studied with metabolic syndrome; only 35 of them developed type 2 diabetes mellitus after a follow-up. Chemerin predicted the incidence of Type 2 diabetes after adjustment for age, sex and body mass index. They concluded that chemerin is a poor predictor of type 2 diabetes mellitus [18].

Salama et al recruited patients for a cross sectional study with chronic kidney disease to assess the relationship of circulating chemerin with atherosclerosis as measured by carotid intima-media thickness. They identified chemerin as a novel adipocytokine regulating and enhancing insulin signaling in fat. Conclusively, they found that chemerin is an independent predictive marker of atherosclerosis in patients with chronic kidney disease [19]. Further review by Shehata et al indicates that chemerin is known to regulate fat formation and metabolism. This is said to play a role in the pathogenesis of metabolic syndrome. They studied a population in Egypt and found that there was an elevated chemerin level seen in obese group when compared with normal controls $(424.20 \pm 61.0$ vs $3000.3 \pm 25, p=0.000)$. Moreso, there is a positive correlation between chemerin and weight $(\mathrm{r}=0.4, p=0.02)$. This proves that serum chemerin levels were significantly increased in obese individuals when compared with lean controls [20].

\section{Possible Pathophysiology}

Though the basic pathophysiology linking chemerin and obesity and type 2 diabetes is not fully clear, a lot of researchers have hypothesized some possible mechanisms. Roman AA et al in their study advocated that adipokines (including chemerin) play a major role in chronic inflammatory conditions and are involved in the regulation of inflammation, adipogenesis, and glucose metabolism. Chemerin does this by its interaction with chemokine -like receptor 1 [13]. Further studies have implicated chemerin in exercabating glucose intolerance which later gives rise to diabetes mellitus by enhancing insulin resistance [11]. It was also found that reduction in insulin levels were associated with increasing chemerin levels [17]. Again, Li et al suggested chemerin enhanced an imbalance in lipid 
metabolism, insulin resistance and altered metabolism of glucose [17]. Xu et al also said that chemerin, being a chemoattractant, is involved in chronic inflammation of adipose tissues in obese conditions. This enables it to play a role in obesity and insulin resistance. Conclusively, Ernestet al found that chemerin exhibited a positive correlation with the aspects of metabolic syndrome (which includes obesity and type 2 diabetes) [22].

\section{Conclusion}

Chemerin may play a role in the pathogenesis of obesity and type 2 diabetes mellitus. More studies and researches have to be done to make conclusions on this subject. Adequate knowledge of this could enhance pharmacological intervention in obese and type 2 diabetic patients, thereby improving patient management.

\section{References}

[1] Conde J, Scotece M, Gómez R, López V, Gómez-Reino JJ, Lago F, Gualillo O (2011). Adipokines: biofactors from white adipose tissue. A complex hub among inflammation, metabolism, and immunity. Biofactors 2011; 37 (6): 413-420.

[2] Gerdes S, Osadtschy S, Rostami-Yazdi M, Buhles N, Weichenthal M, Mrowietz U. Leptin, adiponectin, visfatin and retinol-binding protein-4 - mediators of comorbidities in patients with psoriasis. ExpDermatol. 2012 Jan; 21(1):43-7.

[3] Duvic M, Nagpal S, Asano AT, Chandraratna RA. Molecular mechanisms of tazarotene action in psoriasis. J. Am. Acad. Dermatol. 1997; 37(2Pt 3): S18-24.

[4] Roh SG, Song SH, Choi KC, Katoh K, Wittamer V, Parmentier M, Sasaki S. Chemerin--a new adipokine that modulates adipogenesis via its own receptor. BiochemBiophy ResCommun. 2007; 362 (4): 1013-18.

[5] DuXY, Leung LK. Proteolytic regulatory mechanism of chemerin bioactivity. ActaBiochimBiophys Sin. 2009 Dec; 41(12): 973-979.

[6] Xhou J, Zhang, Chen N, He H, Ye R. Chemerin C9 peptide induces receptor internalization through a clathrinindependent pathway. ActaPharmacol Sin. 2014; 35(5): 653663.

[7] Hah YH, Kim NK, Kim MK, Hur SH, Yoon HJ. Relationship between Chemerin Levels and Cardiometabolic Parameters and Degree of Coronary Stenosis in Korean Patients with Coronary Artery Disease. Diabetes Metab J 2011 Jun; 35(3): 248-254.

[8] Rabe K, Lerhke M, Parhofer KG, Broedl UC. Adipokines and Insulin Resistance. Mol Med. 2008 Nov-Dec; 14(11-12): 741751.
[9] Jung UJ, Choi MS. Obesity and Its Metabolic Complications: The Role of Adipokines and the Relationship between Obesity, Inflammation, Insulin Resistance, Dyslipidemia and Non-alcoholic Fatty Liver Disease. Int J Mol Sci. 2014 Apr; 15(4): 6184-6223.

[10] Yang M, Yang G, Dong J, Liu Y, Zong H, Liu H, Boden G, Li L. Elevated plasma levels of chemerin in newly diagnosed type 2 diabetes mellitus with hypertension. $J$ Investig Med. 2010 Oct; 58(7):883-6.

[11] Ernst MC, Issa M, Goralski KB, Sinal CJ. Chemerin exacerbates glucose intolerance in mouse models of obesity and diabetes. Endocrinology. 2010 May; 151(5):1998-2007.

[12] Coimbra S, Brandão Proença J, Santos-Silva A, Neuparth MJ. Adiponectin, leptin, and chemerin in elderly patients with type 2 diabetes mellitus: A close linkage with obesity and length of the disease. Biomed Res Int2014; Article ID 701915, 8pages.

[13] Roman AA, Parlee SD, Sinal CJ.Chemerin: a potential endocrine link between obesity and type 2 diabetes.Endocrine. 2012 Oct; 42(2):243-51.

[14] Wang D, Yuan GY, Wang XZ, Jia, L, Di L, L. Yang. Plasma chemerin level in metabolic syndrome. Genetics and Molecular Research 2013; 12(4): 5986-5991.

[15] Wang LY, Wei L, Yu HY, Zhang Y, Jia WP. Relationship of serum chemerin to obesity and type 2 diabetes mellitus. . 2009 Feb 3; 89(4):235-8.

[16] Tan X, Li M, Xiao Y. Chemerin correlates obesity and metabolic syndrome and dementia after weight loss in children. Endocrine Abstracts 2016; 41:EP786.

[17] Li Y, Shi B, Li S. Association between serum chemerin concentration and clinical indices in obesity or metabolic syndrome: a meta-analysis. PLOS ONE. 2014; 9(12):e113915.

[18] Robert T, Schwarz F, Fischer-Rosisnsky A, Maurer L, Molig M. Chemerin and prediction of diabetes mellitus type 2 . ClinEndocrinol 2015; 82(6):838-43.

[19] Salama FE, Anass QA, abdelnaem AA, saeed E. chemerin: Abiomarker of cardiovascular disease in diabetic chronic kidney diseases patients. Saudi J Kidney Disease Transpl 2016; 27(5):977-984.

[20] Shehata MA, Ahmed HH, Hameed ER, mokhtar L, Megawer AS. Clinical significance of chemerin in obesity and metabolic syndrome in children. Int Journal of Pharmaceutical and Clinical Research 2015; 7(41):290-295.

[21] Xu H, Barnes GT, Yang Q, Tan G, Yan D, Chon CJ. Chronic inflammation in fat plays a role in the development of obesityrelated insulin resistance. J Clin Invest 2003; 112(12):821-30.

[22] Ernest MC, Sinai CJ. Chemerin:at the crossroads of inflammation and obesity. Trends in Endocrinology and Metabolism 2010; 21(11):660-662. 